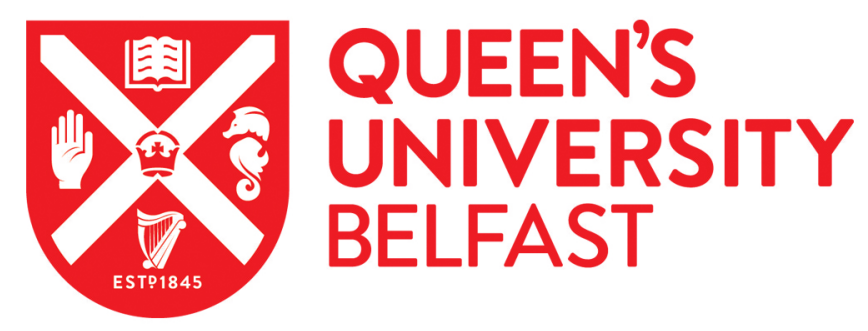

\title{
Time of Flight based diagnostics for high energy laser driven ion beams
}

Scuderi, V., Milluzzo, G., Alejo, A., Amico, A. G., Booth, N., Cirrone, G. A. P., Doria, D., Green, J., Kar, S., Larosa, G., Leanza, R., Margarone, D., McKenna, P., Padda, H., Petringa, G., Pipek, J., Romagnani, L., Romano, F., Schillaci, F., ... Korn, G. (2017). Time of Flight based diagnostics for high energy laser driven ion beams. Journal of Instrumentation, 12, [C03086]. https://doi.org/10.1088/1748-0221/12/03/C03086

\section{Published in:}

Journal of Instrumentation

\section{Document Version:}

Peer reviewed version

\section{Queen's University Belfast - Research Portal:}

Link to publication record in Queen's University Belfast Research Portal

\section{Publisher rights}

(C) 2017 IOP Publishing Ltd and Sissa Medialab slr.

This work is made available online in accordance with the publisher's policies. Please refer to any applicable terms of use of the publisher.

\section{General rights}

Copyright for the publications made accessible via the Queen's University Belfast Research Portal is retained by the author(s) and / or other copyright owners and it is a condition of accessing these publications that users recognise and abide by the legal requirements associated with these rights.

Take down policy

The Research Portal is Queen's institutional repository that provides access to Queen's research output. Every effort has been made to ensure that content in the Research Portal does not infringe any person's rights, or applicable UK laws. If you discover content in the Research Portal that you believe breaches copyright or violates any law, please contact openaccess@qub.ac.uk. 


\section{Time of Flight based diagnostics for high energy laser driven ion beams}

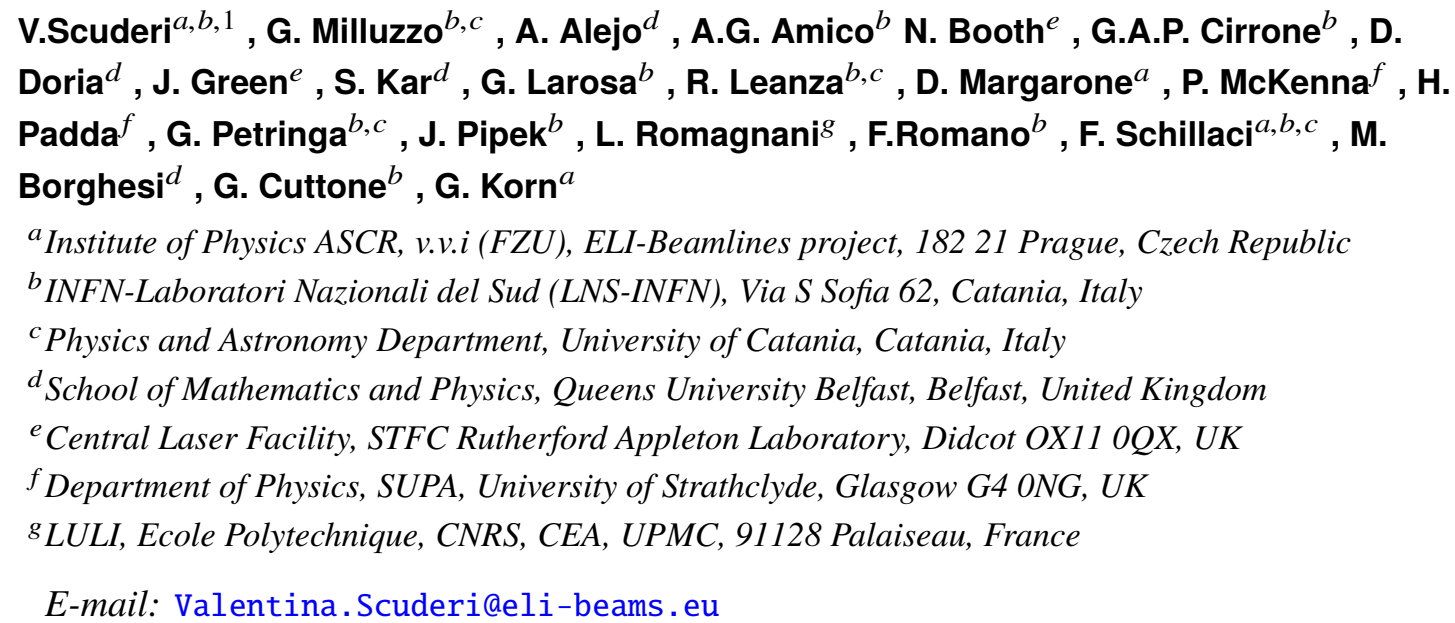

AвSTRACT: Nowadays the innovative high power laser-based ion acceleration technique is one of the most interesting challenges in particle acceleration field, showing attractive characteristics for future multidisciplinary applications, including medical ones. Nevertheless, peculiarities of optically accelerated ion beams make mandatory the development of proper transport, selection and diagnostics devices in order to deliver stable and controlled ion beams for multidisciplinary applications. This is the main purpose of the ELIMAIA (ELI Multidisciplinary Applications of laser-Ion Acceleration) beamline that will be realized and installed within 2018 at the ELI-Beamlines research center in the Czech Republic, where laser driven high energy ions, up to $60 \mathrm{MeV} / \mathrm{n}$, will be available for users. In particular, a crucial role will be played by the on-line diagnostics system, recently developed in collaboration with INFN-LNS (Italy), consisting of TOF detectors, placed along the beamline (at different detection distances) to provide online monitoring of key characteristics of delivered beams, such as energy, fluence and ion species. In this contribution an overview on the ELIMAIA available ion diagnostics will be briefly given along with the preliminary results obtained during a test performed with high energy laser-driven proton beams accelerated at the VULCAN PW-laser available at RAL facility (UK).

KEYwORDs: Ion diagnostics; high energy laser-driven protons; time of flight.

ArXIV EPrint: 1234.56789

${ }^{1}$ Corresponding author. 


\section{Contents}

1 Introduction 1

2 The ELIMAIA diagnostics system 3

3 Results 4

4 Conclusions $\quad 6$

5 Acknowledgments $\quad 7$

\section{Introduction}

The user-oriented ELIMAIA beamline will be installed at the ELI-Beamlines facility in Prague within 2018. The beamline will be dedicated to multidisciplinary and medical applications of ion sources generated by the interaction of $1 \mathrm{PW}$ laser pulse ( $30 \mathrm{~J}, 30 \mathrm{fs})$, with a solid target at high repetition rate, $(1-10 \mathrm{~Hz})$. Furthermore, in a second phase, a PW-class, "long-pulse" (150 J, 150 fs, $1 \mathrm{shot} / \mathrm{min}$ ) will also be available for proof-of-principle experiments in the field of laser plasma acceleration physics. The ELIMAIA beamline is composed of a section dedicated to laser-transport and ion acceleration, consisting of a plasma mirror (PM) chamber and a target interaction chamber (TC) provided with a laser diagnostics system (LD), and a section (an ad hoc "conventional" beamline), named ELIMED, dedicated to transport, selection, diagnostics and dosimetry of the optically accelerated ion beams entirely developed and realized by INFN-LNS in collaboration with ELI-Beamlines. Fig.1 shows a technical layout of the ELIMAIA beamline in the so-called E4 experimental hall of the ELI-Beamlines experimental building.

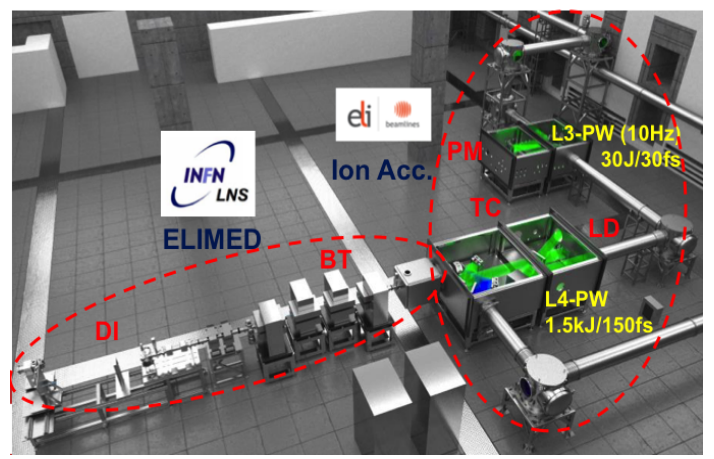

Figure 1. Technical layout of the ELIMAIA beamline in the E4 experimental hall: Plasma Mirror chamber (PM), Laser Diagnostics (LD), Target Chamber (TC), Beam Transport (BT) and Dosimetry and Irradiation (DI). 
The first section of the ELIMED beamline is dedicated to the in vacuum transport, selection and diagnostics of the laser accelerated beam $[1,2]$. A permanent magnet quadrupole system (PMQs) will be placed few $\mathrm{cm}$ downstream the target (i.e. the laser driven ion source) allowing collecting and focusing of the beam energy component of interest [3, 4]. The PMQ system is followed by an energy selector system (ESS), dedicated to ion beam selection in terms of species and energy, based on a magnetic chicane, which consists of four resistive magnet dipoles. According to ion beam optics studies [5], such a solution will allow to select protons up to $300 \mathrm{MeV}$ and carbon ions up to $70 \mathrm{MeV} / \mathrm{n}$ with an energy resolution depending on the central slit aperture going from $5 \%$ up to $20 \%$. The last ELIMED beamline section is in air and is addressed to the relative and the absolute dose measurements of the ion beam, provided also with system for the irradiation of different samples [6]. The main goal of the beamline is to deliver to users down to the irradiation point proton beams which are stable, reproducible and with spatial homogeneity, particle fluence and energy spread not very different to those of conventionally accelerated beams, thus suitable for a broad range of applications. In order to monitor the beam characteristics, in particular energy and angular distribution, ion species and fluence along the beamline, a specific on-line diagnostics system has been developed. The main online detectors system, which will be implemented along the ELIMED beamline, is based on the time of flight (TOF) technique performed using diamond (polycrystalline and single crystal) and silicon carbide detectors. In fact, although never used at such high ion energies (100-MeV level), the TOF technique is so far one of the most established diagnostics method, mainly used in low energy laser-driven beam experiments and low repetition rate laser systems, e.g. through the use of Faraday cups, ion collector rings and also $\mathrm{SiC}$ and diamond detectors as reported in literature [7-10], hence offering the possibility to measure online energy cutoff, fluxes, ion species and obtain a shot-to-shot control of the beam features. The ELIMAIA beamline will be provided with two TOF diagnostics sections. Indeed, in view of the high laser repetition rate foreseen at ELIMAIA, up to $10 \mathrm{~Hz}$, the on-line TOF based diagnostics will allow monitoring the beam each laser shot and measuring online the beam parameters. One detector will be placed about $2 \mathrm{~m}$ downstream the target, after the PMQ system and just before the energy selector injection point. This first diagnostics stage, consisting of a diamond detector mounted at $0^{\circ}$ along the beam trajectory, will allow to monitor online the stability and reproducibility of the proton/ion beam parameters, in particular measuring the maximum energy cutoff, the beam flux and the energy spectrum of the focused beam shot to shot just before the selection section. Another diamond detector will be placed at the end of the in vacuum beamline along the beam axis, about $9 \mathrm{~m}$ downstream the target, after the ion selection in energy. This second diagnostics stage will allow to measure energy distribution, fluence and energy bandwidth of the selected proton beam for the different energy ranges of interest. The online beam parameter characterization given by the TOF measurement at both the two flight paths, will provide crucial information for the transport optimization, giving also the possibility to tune in "real time" and adjust the PMQs and the ESS parameters according to the required beam specification. The ELIMAIA diagnostics system performances have been tested using the TOF technique with the high energy laser-driven proton beam generated by the Petawatt Vulcan laser at the Rutherford Appleton Laboratory (RAL). Preliminary results of such test will be presented in this contribution along with a brief description of the developed analysis procedure. 


\section{The ELIMAIA diagnostics system}

Due to the extremely peculiar features of laser-driven ion beams available today, such as the short pulse duration (0.1-1 ns near the source), the peak current $\left(10^{10}\right.$ to $\left.10^{12} \mathrm{p} / \mathrm{pulse}\right)$ and the very high dose-rates in a single pulse $\left(10^{9} \mathrm{~Gy} / \mathrm{min}\right)$ compared to those of the conventionally accelerated beams [11-13], innovative techniques and devices for beam diagnostics have to be developed since a robust online diagnostics represents one of the crucial steps towards multidisciplinary applications of such beams. Diamond and silicon carbide detectors show interesting characteristics [8-10] in TOF measurements of high energy laser-driven proton beams, such as good time and energy resolution, radiation hardness in order to support fluxes up to $10^{9} \mathrm{p} / \mathrm{bunch}$ and low capacitance which makes them particularly attractive for timing measurements of high intensity particle bunch at high repetition rate. The ELIMAIA diagnostics system consists of chemical vapour deposited polycrystalline (pDD) and single crystal diamond (sDD) detectors and SiC detectors with different surface sizes, between $2 \mathrm{~mm}$ and $5 \mathrm{~mm}$ diameter, and thicknesses, between $20 \mu \mathrm{m}$ and $500 \mu \mathrm{m}$. In particular, a thin (about $100 \mu \mathrm{m}$ ) pDD will be used to measure the TOF signal at about 2 $\mathrm{m}$ downstream the source, i.e after the PMQ system, and a thick (about $500 \mu \mathrm{m}$ ) sDD will be used to measure the TOF signal of the proton group selected by the energy selection, downstream the in-vacuum beamline at about $9 \mathrm{~m}$ from the source. Indeed, according to ion beam optics studies [4], about $20 \%$ of the particles generated at the source, i.e. about $10^{9}$ in the worse case, will be transmitted through the focusing system with a broad energy distribution, therefore a low capacitance, fast detector suitable for high intensity loss measurements, as a thin pDD, will allow to measure the TOF of the whole proton group with a reasonably good time resolution with a 2 $m$ flight path even for the fastest energy component. On the other hand, downstream the selection system, as an example, for the $60 \mathrm{MeV}$ proton transport and selection a minimum proton number of about $10^{7}$ per bunch with $20 \%$ energy spread is expected [5], hence a sDD, thanks to an excellent signal-to-noise ratio, will allow to measure the narrow energy proton distribution selected.

A single crystal diamond detector (sDD), $3 \times 3 \mathrm{~mm}^{2}$ and $500 \mu \mathrm{m}$ thick, and polycrystalline diamond detector (pDD), $2 \times 2 \mathrm{~mm}^{2}$ and $100 \mu \mathrm{m}$ thick, both supplied by the CIVIDEC company [14], have been recently tested in TOF measurements of the high energy laser-driven proton beams generated by the Petawatt Vulcan laser at the Rutherford Appleton Laboratory (RAL). The VULCAN laser is a neodymium glass laser operating at a central wavelength of $1053 \mathrm{~nm}$, delivering about $650 \mathrm{~J}$ in a time pulse of about $500 \mathrm{fs}$, able to accelerate protons up to $60 \mathrm{MeV}$ [15]. The goal of the experiment was to measure the temporal evolution signal of the high energy optically accelerated proton beam, test the developed TOF diagnostics with diamond detectors for the first time with high-energy (several tens of $\mathrm{MeV}$ ) laser-driven proton beam and extract from the TOF signal the energy cut-offs, the energy distribution and the correspondent fluence. The target used for the experiment was a flat $25 \mu \mathrm{m}$ thick $\mathrm{Al}$ foil. The detectors were placed both in the forward and in the backward direction at about $10^{\circ}$ with respect to the target normal direction and at different distances, i.e. flight paths, from the target between about $1 \mathrm{~m}$ and $4 \mathrm{~m}$. In order to compare the energy distribution and the fluence extracted from the TOF signal a Thomson Parabola (TP) spectrometer coupled with imaging plate detectors and CR39 nuclear track detectors were used in the backward direction measurements. A schematic layout of the experimental setup is shown in fig.2.

TOF signals were stored by a $2 \mathrm{GHz}$ Le Croy digital oscilloscope terminated with a $50 \mathrm{Ohm}$ 


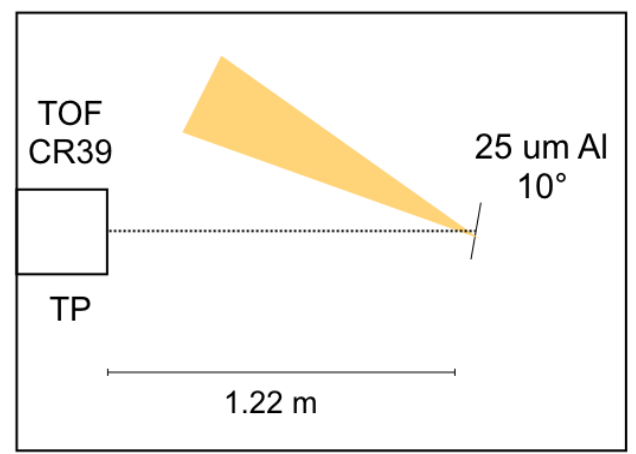

Figure 2. A sketch of the experimental setup.

load impedance. Diamond detectors were provided with an integrated HV circuit consisting of a simple $\mathrm{RC}$ circuit $(\mathrm{R}=2 \mathrm{M} \Omega, \mathrm{C}=100 \mathrm{nF})$ optimized to compensate possible detector discharges and a stable charge collection. A power supply module has been used to provide a bias voltage of 200 $\mathrm{V}$ to the $\mathrm{pDD}$ and $500 \mathrm{~V}$ to the sDD.

\section{Results}

A typical TOF signal acquired with the $\mathrm{pDD}$ at about $1 \mathrm{~m}$ from the target in the backward direction is shown in fig. 3. A $50 \mu \mathrm{m} \mathrm{Al}$ absorber was used to shield the detector and to stop $2 \mathrm{MeV}$ protons. As one can clearly see in fig. 3 , three peaks can be identified: the photopeak originating from the

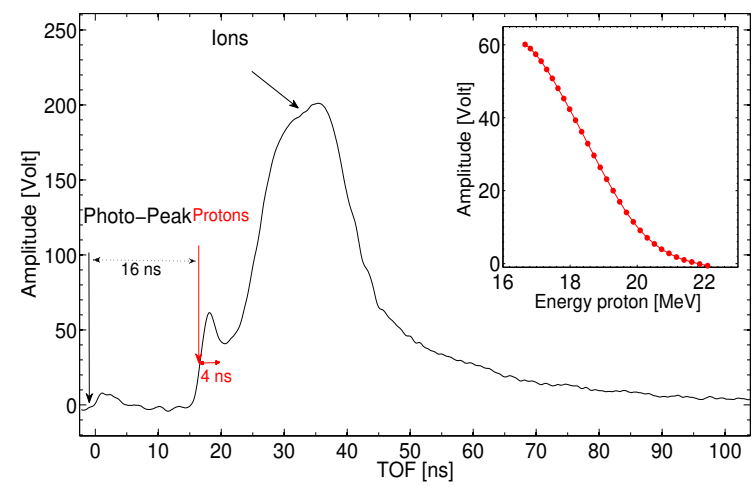

Figure 3. A TOF signal acquired with the pDD @ $1.22 \mathrm{~m}$ downstream the target. The inset shows the incident kinetic energy calculated in the range corresponding to the faster proton peak.

UV and X-ray laser-plasma radiation followed by a tail due to fast plasma electrons ( $0-8 \mathrm{~ns})$, the peak generated by the fastest proton group (16-22 ns) and a broad peak arising from different ion species and charge state contribution, typically present in the target as contaminants, together with the slow proton component (22-47 ns). The incident proton kinetic energy can be calculated from the measured TOF signal given the flight path, $L$. The measured TOF has to be corrected for the 
distance travelled from the source to the detector by the UV and X-rays using the formula:

$$
t=T O F+\frac{L}{c}
$$

where $c$ is the speed of light and $t$ is the TOF corrected for the photon flight path. Then, the incident proton kinetic energy can be obtained using the corrected TOF, $t$, by the well-known definition:

$$
E_{k i n}=(\gamma-1) M_{p} c^{2} \quad \gamma=\frac{1}{\sqrt{\left(1-\beta^{2}\right)}} \quad \beta=\frac{L}{c t}
$$

where $M_{p}$ is the rest mass of proton and $L$ is the flight path (i.e. the target-diamond detector distance). As one can see in fig.3, the peak arising from the fast protons is well separated by the slow ion contribution and rise time of $1.5 \mathrm{~ns}$ can be observed. The inset in fig. 3 shows the incident kinetic energy calculated taking into account the relativistic correction from the measured TOF in the range between $16 \mathrm{~ns}$ and $22 \mathrm{~ns}$, corresponding to the high energy proton peak. The shortest TOF measured (16 ns) indicates maximum proton energy of about $22 \mathrm{MeV}$. Moreover, as one can see from fig.3, due to the diamond low sensitivity to the plasma visible and soft-UV radiation and the fast time response, a short photopeak with a temporal decay of about $5 \mathrm{~ns}$ and a rise time less than 1 ns can be observed. Thanks to the pDD performances it has been possible to clearly disentangle the proton energy cutoff and the high energy component from the photopeak even at about $1 \mathrm{~m}$ from the laser-driven source. Taking into account that the energy cutoff ultimately expected for the high energy proton beam accelerated along the ELIMAIA beamline will be between $60 \mathrm{MeV}$ and 100 $\mathrm{MeV}$ corresponding to a TOF ranging between $16 \mathrm{~ns}$ to $20 \mathrm{~ns}$ at $2 \mathrm{~m}$ from the source, the obtained results confirm that using the TOF technique with the pDD it will be possible to discriminate the photopeak and the maximum proton energy at the first diagnostics section. The broad signal in the TOF range between $22 \mathrm{~ns}$ and $47 \mathrm{~ns}$ in fig. 3 is due to the contribution of light ions, mainly carbon and oxygen together with contaminants in the target, together with the low energy proton group. The accelerated ion contaminants and their energies have been identified thanks to simultaneous measurements performed using the TP spectrometer. The fluence and the energy distribution, $\mathrm{dN} / \mathrm{dEd} \Omega$, in the TOF region where only the high energy proton contribution is present can be extracted by performing a deconvolution of the time evolution signal of the fast proton peak using a fitting procedure based on the well-known Maxwell-Boltzmann shifted functions. The proton flux per solid angle can be, then, obtained from the fitted signal amplitude using the formula reported in $[8,16,17]$. However, considering the high proton energies measured in order to obtain the proton flux from the fitted signal amplitude, the energy loss deposited by the fast protons in the detector active thickness, much thinner than the fast proton range in the diamond, has to be calculated and considered. The complete analysis performed on the TOF spectra will be reported elsewhere. The correction for the effective energy loss for high incident kinetic energy protons will be particularly important to accurately extract the proton flux from the TOF spectra at the typical energies expected at the ELIMAIA beamline. In fig. 3 in the TOF range between $22 \mathrm{~ns}$ and $47 \mathrm{~ns}$ due to the overlap of different ion contribution it is not possible to directly extract the incident proton kinetic energy from the measured TOF using the formula 3.2. Therefore, in this case the discrimination of the single ion species contribution from the TOF signal and, also, the reconstruction of the ion energy distribution result more difficult and a more complex analysis is required. In this case, the 
TOF signal deconvolution can be carried out coupling the TOF signal with the contribution of the different ion species extracted from the TP parabola spectrometer measurements. Nevertheless, the first diagnostics section along the ELIMAIA beamline is placed downstream the PMQ focusing system, at this stage an ion component might still be transported together with the focused proton beam therefore the TOF spectrum will result in a signal due to the overlap of different ion species. In this case, the TOF signal deconvolution will be improved coupling the TOF measurements with a TP parabola spectrometer designed and developed at ELI-Beamlines. On the other hand, the second diagnostics section is placed downstream the in vacuum beamline after the energy selection system. At the end of the in-vacuum beamline only a selected proton beam component will be transported, therefore, the TOF signal will be only ascribable to protons with the selected energy, thus the analysis procedure to extract the energy spectrum will be more straightforward as shown in fig. 3 for the spectrum region where only the fast proton contribution is present. In view of the ELIMAIA diagnostics system development, the TOF diagnostics system described in this work will be improved using passive and also thin active absorbers in order to stop the heavier ions, detect only the fast protons and reconstruct the contribution of the stopped ions. Furthermore, an array of diamond detectors will be assembled and tested in order to provide also measurement of the beam energy spectrum as a function of the emission angle from the source.

\section{Conclusions}

The on-line diagnostics system developed for the laser driven ion beamline at ELI-Beamlines (ELIMAIA) will be based on the time evolution measurement of the optically accelerated ion beams using the well-known TOF technique to extract the ion energy and flux. The ELIMAIA diagnostics system consists of thin diamond detectors. Indeed, among the different detectors so far used for low energy laser-driven TOF measurements, as for instance Faraday cup and ion collector, diamond detectors thanks to higher time and energy resolution, radiation hardness and low photon detection sensitivity are particularly indicated for high energy intense laser-driven ion beam measurements at high repetition rate in harsh plasma environment. The TOF measurements performed with a thin polycrystalline diamond using high energy proton beams accelerated by the VULCAN laser at RAL (UK) indicated that thanks to the diamond performances it will be possible to monitor online the laser-driven proton beam parameters, such as energy cutoff and flux, for the high proton energy expected (up to $100 \mathrm{MeV}$ ) even at a very short flight path (i.e. about $2 \mathrm{~m}$ from the source). Moreover, thanks to the diamond detector excellent time resolution it will be possible to measure the energy bandwidth of the proton beam selected downstream the beamline. In such a way, a shot to shot monitoring provided by the TOF diagnostics, coupled also with the TP spectrometer, will allow tuning the laser parameters and optimizing the proton transport from the focusing system through the energy selection in order to deliver a stable proton beam to the users to whom such proton beam will be delivered to explore a broad range of multidisciplinary applications. 


\section{Acknowledgments}

This work has been performed within the ELIMED activities supported by the V committee of INFN (Italian Institute for Nuclear Physics), by the MIUR (Italian Ministry of Education, Research and University), by the ELI-Beamlines Contract no S14-187 under under CZ.02.1.01/0.0/0.0/15 _ 008/0000162 through the European Regional Development Fund, by the Ministry of Education, Youth and Sports of the Czech Republic (National Program of Sustainability II Project No. LQ1606) and by the EPSRC (grant n. EP/K022415/1).

\section{References}

[1] G.A.P. Cirrone et al., Transport and dosimetric solutions for the ELIMED laser-driven beam line, Nucl. Instrum. Meth. A 796 (2015) 99.

[2] G.A.P. Cirrone et al., Design of the prototype of a beam transport line for handling and selection of low energy laser-driven beams, Appl. Sci. 5 (2015) 427.

[3] F. Schillaci et al., Design of the prototype of a beam transport line for handling and selection of low energy laser-driven beams, Nuclear Instruments and Methods in Physics Research A 837 (2016) 80

[4] F. Schillaci et al., Design of the ELIMAIA ion collection system, JINST 10 T12001 (2015)

[5] F. Schillaci et al., Design of a large acceptance, high efficiency energy selection system for the ELIMAIA beam-line, JINST 11 P08022

[6] F. Romano et al., The ELIMED transport and dosimetry beamline for laser-driven ion beams, Nuclear Instruments and Methods in Physics Research A 829(2016)153-158

[7] D. Margarone et al, Diamond detectors for characterization of laser-generated plasma, Rad Eff Def. Solids 163, 2008, 463

[8] D. Margarone et al., Full characterization of laser-accelerated ion beams using Faraday cup, silicon carbide, and single-crystal diamond detectors, J. Appl. Phys. 109, 103302 (2011)

[9] M. Marinelli et al., Analysis of laser-generated plasma ionizing radiation by synthetic single crystal diamond detectors, Applied Surface Science 272 (2013) 104-108.

[10] G. Bertuccio et al., Silicon carbide detector for laser-generated plasma radiation, Applied Surface Science 272 (2013) 128-131

[11] E.L. Clark et al., Measurements of Energetic Proton Transport through Magnetized Plasma from Intense Laser Interactions with Solids, Physical Review Letters 84 (2000) 670.

[12] A. Maksimchuk, S. Gu, K. Flippo, D. Umstadter, Y.V. Bychenkov, Forward Ion Acceleration in Thin Films Driven by a High-Intensity Laser, Physical Review Letters (2000) 8.

[13] R.A. Snavely et al., Intense High-Energy Proton Beams from Petawatt-Laser Irradiation of Solids, Physical Review Letters (2000) 85.

[14] CIVIDEC https://cividec.at

[15] C.N Danson et al., Vulcan Petawatt an ultra-high-intensity interaction facility, Nuclear Fusion, Volume 44, Number 12

[16] G. Milluzzo et al., Laser-accelerated ion beam diagnostics with TOF detectors for the ELIMED beam line, JINST Volume 12, February 2017 
[17] G. Milluzzo et al., TOF technique for laser-driven proton beam diagnostics for the ELIMED beamline, JINST Volume 12, March 2017 